www.jmscr.igmpublication.org

Index Copernicus Value: 79.54

ISSN (e)-2347-176x ISSN (p) 2455-0450

crossrefDOI: https://dx.doi.org/10.18535/jmscr/v7i3.161

Journal Of Medical Science And Clinical Research

IGM Publication

An Official Publication of IGM Publication

\title{
Cytopathological Study of Salivary gland Tumor of Patients, Attending in Tertiary Care Hospital at Muzaffarpur, Bihar
}

\author{
Authors \\ Dr Mahesh Prasad ${ }^{1}$, Dr Manoj Kumar ${ }^{2}$ \\ ${ }^{1}$ Associate Professor, Department of Pathology, Sri Krishna Medical College, Muzaffarpur \\ ${ }^{2}$ Professor and HOD, Department of Pathology, Sri Krishna Medical College, Muzaffarpur \\ Corresponding Author
}

Dr Mahesh Prasad

Associate Professor, Department of Pathology, Sri Krishna Medical College, Muzaffarpur

\begin{abstract}
Objectives: Salivary glands tumor are morphologically and clinically diverse group of neoplasm which may present with significant diagnostic and management challenges because of their relative frequency, limited amount of pretreatment information available and wide variety of biological behavior with different pathological lesions. The aim of present study was to know the incidence, spectrum of morphological features of Salivary glands Tumor and their clinical correlation with that of histopathological findings.

Materials and Methods: In the diagnosis of salivary gland lesions, FNAC has gained the popularity as diagnostic tool due to its low cost and safe procedure with minimal risk to the patient. A total of 76 patients suffering from Salivary gland swelling were attending in ENT and Surgery OPD was included in the study. All patients were evaluated by detailed history, clinical examinations, and by routine tests. FNAC will be performed from different sites of salivary glands swelling using 10m1 disposable syringe and 22/23 gauge needle. Aspirated samples were smeared on glass slide and air dried, which are stained with Giemsa stain and wet smear is fixed in 95\% ethyl alcohol and are stained with Papanicolaou stain. After surgery 32 salivary tissues were received in for histopathological evaluation. After ensuring adequate tissue fixation, tissue slices were taken and processed. Microsections of 5 microns thickness were taken onto glass slides and stained by standard Hematoxylin and Eosin stains. After mounting and labeling smears were seen under oil immersion microscope.

Results: Out of 76 patients, 44 (57.9\%) patients were females and $32(42.1 \%)$ patients were male. Male to female ratio was 0.72:1. Out of 76 cases of salivary gland lesion, Parotid gland 57 ( $75 \%$ ) was the most common gland involved followed by submandibular gland 16 (21.05\%) cases, minor salivary gland in 2 (2.63\%) cases and 1 (1.32\%) cases was lingual gland. Malignant lesions are more common in $7^{\text {th }}$ decade followed by $5^{\text {th }}$ and $4^{\text {th }}$ decade. In Histocytological evaluation it is observed that inflammatory lesions was found in 48 cases (63.15\%) and were the most common cytological findings followed by benign lesion in $26(34.21 \%)$ cases and malignant lesions in 2 (2.63\%) cases. Among benign tumor pleomorphic adenoma was the most common findings observed, while among malignant tumor 1 case was of Adenoid cystic carcinoma and 1 case was mucoepidermoid carcinoma was found. Total histological and cytological correlation is available in 32 cases. The sensitivity, specificity and diagnostic accuracy is $90.2 \%, 97.6 \%$, and $90.2 \%$, respectively.

Conclusion: Salivary glands tumor is common, account approximate $6 \%$ of head and neck tumor but exhibit a wide variety of microscopic appearance even within one particular lesion. Accurate diagnosis is essential as salivary gland neoplasm have diverse clinical and prognostic outcome. The idea of lesion cannot be resolved on clinical examination only, so pathological examination is required for distinct conclusion.
\end{abstract}

Keywords: salivary gland tumor, FNAC, malignant tumors, pleomorphic adenoma. 


\section{Introduction}

The salivary gland system includes three pairs of major glands parotid, submandibular and sublingual and many minor glands in the sub mucosa of oral cavity. Minor salivary glands can be found in the lips, floor of mouth, gingival, cheek, hard and soft palates, tongue, tonsillar areas and oropharynx. The lesions of salivary glands commonly create clinical problems. They range from non neoplastic lesions to benign and malignant tumors. Fine needle aspiration cytology $(($ FNAC $))$ is an accepted, sensitive and specific diagnostic modalities for of the salivary gland lesions. Salivary gland cancer is rare, with $6 \%$ of head and neck tumors forming in the salivary glands, the majority in the parotid glands.

Salivary gland Tumors is usually found in patients between the ages of 18 and 68 years (mean: 43 years) with slight overall female predominance, male to female ratio of $1: 1.8$, this finding is similar with the other reports. The SGTs are classified by (WHO Classification of Epithelial Salivary glands tumor) Benign Epithelial tumor (Pleomorphic adenoma, Myoepithelioma and Basal cell adenoma), Malignant Epithelial (tumor Acinic cell carcinoma, Mucoepidermoid carcinoma and Squamous cell carcinoma), Nonepithelial or Mesenchymal tumor, Malignant Lymphoma, Secondary metastatic tumors, Unclassified tumor, Tumor like lesion like sialadenosis and various cysts

Salivary glands tumors most often present as painless enlarging masses usually located in the parotid glands and are benign mostly. Tumors can occur in both the major and minor salivary glands. $80 \%$ of major salivary gland tumors occur in the parotid glands, while most minor salivary tumors are located in the palate. In the parotid glands, 20$25 \%$ of the tumors are malignant. This rises to $40 \%$ for the submandibular glands, and more than $90 \%$ of sublingual gland tumors are malignant. In both the major and minor salivary glands, the commonest type of benign tumor is pleomorphic adenoma. Pleomorphic adenoma, also called benign mixed tumor, can occur in the deep lobe of the parotid gland with extension into the Para pharyngeal space, which makes it the commonest type of Para pharyngeal space tumor constituting $40 \%$ of tumors. The diagnostic efficacy of FNAC is good with large study series and showing sensitivity up to $85 \%$ and specificity up to $99 \%$.

\section{Materials and Methods}

Present study was conducted in the Department of Pathology, Sri Krishna Medical College, Muzaffarpur, with the help of Department of E.N.T and Surgery, during the period of January 2016 to December 2018. A total of 76 patients attending ENT and Surgery O.P.D or IPD presented with salivary gland swelling, were included in the study. All the patients were evaluated by detailed history, clinical examination, and routine hematological and radiological investigations. FNAC will be performed from different sites of salivary glands swelling using 10m1 disposable syringe and 22/23 gauge needle without local anesthesia. Aspirated samples were smeared on grease free glass slide and air dried, which are stained with Giemsa stain and wet smear is fixed in 95\% ethyl alcohol are stained with Papanicolaou stain. After surgery 32 salivary tissues were received for histopathological evaluation. After ensuring adequate tissue fixation, tissue slices were taken and processed. Microsections of 5 microns thickness were taken onto glass slides and stained by standard Hematoxylin and Eosin stains. After mounting and labeling smears were seen under oil immersion microscope.

\section{Results}

Out of 76 patients, $44(57.9 \%)$ patients were females and $32(42.1 \%)$ patients were male. Male to female ratio was $0.72: 1$.

Out of 76 cases of salivary gland lesion, Parotid gland $57(75 \%)$ was the most common gland involved followed by submandibular gland 16 $(21.05 \%)$ cases, minor salivary gland in $2(2.63 \%)$ cases and $1(1.32 \%)$ cases was lingual gland. 
Malignant lesions are more common in $7^{\text {th }}$ decade followed by $5^{\text {th }}$ and $4^{\text {th }}$ decade. In Histocytological evaluation it is observed that inflammatory lesions was found in 48 cases $(63.15 \%)$ and were the most common cytological findings followed by benign lesion in 26 (34.21\%) cases and malignant lesions in $2(2.63 \%)$ cases. Among benign tumor pleomorphic adenoma was the most common findings observed, while among malignant tumor 1 case was of Adenoid cystic carcinoma and 1 case was mucoepidermoid carcinoma was found.

Total histological and cytological correlation is available in 32 cases. The sensitivity, specificity and diagnostic accuracy is $90.2 \%, 97.6 \%$, and $90.2 \%$, respectively.

Table Shows Histopathological Evaluation of Salivary Glands Tumors.

\begin{tabular}{|c|c|c|c|}
\hline Diagnosis & Histopathological Evaluation & Total no. of patients & Percentage \\
\hline $\begin{array}{l}\text { Inflammatory Lesion } \mathrm{n}=48 \\
(63.15 \%)\end{array}$ & Inflammatory & 48 & 63.15 \\
\hline \multirow{4}{*}{$\begin{array}{ll}\text { Benign } & \text { Lesion } \\
n=26(34.21 \%) & \end{array}$} & Pleomorphic Adenoma & 20 & 26.31 \\
\hline & Warthims Tumors & 3 & 3.94 \\
\hline & Benign Cystic Lesion & 2 & 2.63 \\
\hline & Benign Lesion & 1 & 1.31 \\
\hline \multirow{2}{*}{$\begin{array}{ll}\text { Malignant } & \text { Lesion } \\
n=2(2.63 \%) & \end{array}$} & Adenoid Cystic Carcinoma & 1 & 1.31 \\
\hline & Mucoepidermoid Carcinoma & 1 & 1.31 \\
\hline
\end{tabular}

\section{Discussion}

The precise histological diagnosis is made by examination of the post-operative specimen. FNAC is the excellent techniques for the diagnosis of the salivary glands although this has its limitations. Studies have demonstrated that repeated fine needle aspiration is associated with increased chance of diagnosing the lesion. In the diagnosis of salivary gland lesions, FNAC has gained the popularity as diagnostic tool due to its low cost and safe procedure with minimal risk to the patient and help to the surgeons for treatment. In present study, it is found that out of 76 patients, females (44 cases, 57.9\%) were more affected than males (32 cases, $42.1 \%$ ) belonging to $3^{\text {rd }}$ decade and male to female ratio was 0.72:1. A study in Iran reported a mean age of 40 years for the occurrence of salivary gland tumors. Generally, this study showed that there were more females who reported to hospital with salivary gland conditions than males at a ratio of 1.8:1. Al Khateeb et al in Jordan and Zhao et al in India reported similar trends. However, the studies by Chidzong in Zimbabwe and Nitin in India had higher numbers of males compared to females. The higher number of females may be due to the fact that generally Indian women tend to be more immunogenicity due to poor hygienic and economic conditions. There was no any male or female differentiation for benign or malignant lesions. However salivary gland lesions are more common in females.

In the present study, most common non-neoplastic lesion was sialadenitis followed by cystic lesion and most of the non-neoplastic lesions involved the parotid. Dilip $\mathrm{K}$ et al studied the similar observation in his study.

In present study among benign lesions pleomorphic adenoma (26.31\%) is the most common lesion which is consistent with above studies. Pleomorphic adenoma was the most common benign tumor, with slight female dominance occurring mainly in the third decade of life. It most commonly affects the parotid gland, cytology of the tumor shows a bland epithelial cell in aggregates and sheets and fragments of the chondromyxoid fibrillary stroma.

In present study Warthin's tumour, the second most common $3.94 \%$ benign tumor occurs mainly in the fourth to sixth decade, with a male preponderance. Faur A et al and Lima SS et al also find the similar findings in their studies.

Various authors have reported that the incidence of malignant tumors ranged from $15 \%$ to $32 \%$. In 
present study it is $2.63 \%$, which was low due to low sample size and short duration of study. Classifying the salivary gland lesions into nonneoplastic, benign and malignant neoplasm may guide the clinician to make correct therapeutic decisions as to which patient requires further investigation, medical treatment or excision. In malignant tumor $1(1.31 \%)$ case was of Adenoid cystic carcinoma and $1(1.31 \%)$ case was mucoepidermoid carcinoma was found. Similar to other studies of Schoeman BJ et al and Eglis K et al. However, few more studies also document it to be the most common salivary gland tumor. Cytological features of salivary gland tumors can often overlap, hence the final diagnosis must always take into account like cellular, stroma and clinical features.

Comparative analysis of sensitivity and specificity of fine needle aspiration cytology with other studies was as Paik et al has studied sensitivity and specificity of $89.7 \%$ and $96.3 \%$ respectively. Laurie et al has studied sensitivity and specificity of $66 \%$ and $100 \%$ respectively. Canan et al has studied sensitivity and specificity of $66 \%$ and $100 \%$ respectively. Das et al has studied sensitivity and specificity of $94.3 \%$ and $75 \%$ respectively. In present study sensitivity and specificity of $90.2 \%$ and $97.6 \%$ was observed respectively.

FNAC is a reliable examination procedure that provides valuable information for the preoperative diagnostic work-up and alerts the surgeon to the possible presence of malignancy. As the complication rate logically increases with the degree of invasiveness of the surgical procedure and correctly inform the patient about the type of surgery that will be performed, the need for lymph node dissection and the possibility of nerve sacrifice.

\section{Conclusion}

Awareness of the therapeutic implications and limitations of the cytological interpretation amongst both the clinicians and the cytopathologists should enable FNAC to its best advantage. Although FNAC of the salivary gland tumors has a high diagnostic accuracy, it can further be improved by a wider sampling and ultrasound guided aspirations.

\section{References}

1. S. R. Orell, "Diagnostic difficulties in the interpretation of fine needle aspirates of salivary gland lesions: the problem revisited," Cytopathology, vol. 6, no. 5, pp. 285-300, 199

2. Cajulis RS, Gokaslan ST, Yu GH, FriasHidvegi D. Fine needle aspiration biopsy of the salivary glands, a five year experience with emphasis on diagnostic pitfalls. Acta Cytol 1997; 41: 1412- 1420.

3. A. R. Arshad, "Parotid swellings: report of 110 consecutive cases," Medical Journal of Malaysia, vol. 53, no. 4, pp. 417-422, 1998.

4. P. M. Speight and A. W. Barrett, "Salivary gland tumours," Oral Diseases, vol. 8, no. 5, pp. 229-240, 2002.View at Publisher • View at_Google Scholar View at Scopus.

5. Ahrnad S, Lateef $M$, Ahmad R. Clinicopathological study of primary salivarygland tumors in Kashmir. JK practitioner. 2002;9(4):231-33.

6. Juan Rosai- Major and minor salivary glands. In: Michael Houston ed. Rosai and Ackarman's Surgical Pathology. 9th ed. Elsevier India Private Limited, 2004:873-916.

7. Elhosseiny A. Salivay glands. In: Koss LG, Melamed MR, eds. Koss Diagnostic Cytology and its Histopathological basis. 5th ed. Lippincott Williams \& Wilkins; 2006:12291261. 12. Gandhi SH, Purohit TM.

8. Subhashraj K. Salivary gland tumors: A single institution experience in India. $\mathrm{Br} \mathrm{J}$ Oral Maxillofac Surg. 2008;46:635-8. [PubMed]

9. Harari PM; Connor NP; Cai Grau (12 June 2009). Functional Preservation and Quality of Life in Head and Neck Radiotherapy. Springer Science and Business Media.

10. Kalburge JV, Kalburge V, Latti B, Kini Y. Salivary gland tumors: Clinicopathologic analysis of 73 cases. J Cranio Max Dis. 2014; 